\title{
Primary breast leiomyosarcoma: case report and literature review
}

\author{
Fatima Zahra EL M'rabet ${ }^{1,2^{*}}$, Hanane El kacemi ${ }^{1}$, Omar El Mesbahi ${ }^{2}$, Khalid El Hassouni $^{1}$, \\ Brahim khalil El Gueddari $^{1}$
}

${ }^{1}$ Radiotherapy unit, National Institute of Oncology, Rabat, Morocco; ${ }^{*}$ Corresponding Author: fazoumed@hotmail.com

${ }^{2}$ Departement of medical oncology, university hospital Hassan II, Fez, Morocco.

Received 4 April 2011; revised 16 May 2011; accepted 29 May 2011.

\begin{abstract}
PURPOSE: Leiomyosarcoma of the breast is extremely rare. To date, the factors that are predictive of patient prognosis have not been identified. To clarify the nature of leiomyosarcoma of the breast, and also to establish the proper treatment strategy, we report this case We report this case while discussing The Clinical presentation, diagnosis, therapy and pathologic feature. INTRODUCION: Sarcomas comprise less than $1 \%$ of all primary breast neoplasms and only a minority of these are leiomyosarcomas. There were only 35 cases being reported in English literature. The mainstay of treatment is surgical excision with clear margins and longterm followup is essential. Its prognosis is better compared to other breast sarcoma. CASE PRESENTATION: We report a case of primary leiomyosarcoma of the breast occurring in young female patient which was successfully treated by surgery and radiotherapy. CONCLUSION: According to literature data, primary leiomyosarcoma is characterized by a better prognosis compared with other breast sarcomas, To date, the factors that are predictive of patient prognosis have not been identified. The rarity and diagnostic difficulty imposed a multidisciplinary approach.
\end{abstract}

Keywords: leiomyosarcoma; Breast; Immunohistochemistry; Mastectomy

\section{INTRODUCTION}

Sarcomas of the breast account for less than $1 \%$ of all malignant tumors of the breast. Leiomyosarcomas belong to a less common subgroup (5\% to $10 \%$ ) of sarcomas of the breast [1]. There were only 35 cases being reported in English literature. Confirming the diagnosis on either immunohistochemistry is important as it is sometimes difficult to differentiate these pathologically from poorly differentiated sarcomatoid carcinomas. To date, the factors that are predictive of patient prognosis have not been identified. [2]

In this report, we describe case with this uncommon Tumor, which was treated successfully in The Institute of National Oncology Rabat, Morocco.

\section{CASE REPORT}

A 40-year-old female patient, without any significant history, who presented a left breast lump, it has been present for 17 months. There was no other breast complaint and no family history of breast cancer. Physical examination found a tumor of $6 \mathrm{~cm}$ in diameter in the lower inner quadrant of her right breast with ipsilateral axillary lymph node. The left breast was unremarkable. Mammography showed a dense, well circumscribed mass with little evidence of local invasion and the lump was easily detectable by ultrasound. An incisional biopsy was performed, the histological exam showed a sarcomatous proliferation, Immunohistochemically, the tumor cells were diffusely and strongly positive for $\alpha$-smooth muscle actin and vimentin (Figure 1), while negative for cytokeratin, CD34 and S-100 protein, which concludes with primary breast leiomyosarcoma .

Investigations including Liver echography, chest scan and bone scan were showed no evidence of another primary tumor or metastatic disease. The patient underwent a radical mastectomy with lymph node dissection. Additional immunohistochemical examen routinely performed in breast carcinomas (oestrogen, progesteron receptors, Her 2) also proved to be negative. Tumor cells showed strong positivity for vimentin, desmin and smooth muscle actin (SMA). Axillary lymph nodes were free of metastases Axillary. Subsequently the patient had received adjuvant radiotherapy to 50 Gray, the patient is in good control until now. A follow-up examination revealed no evidence of distant metastasis or local recurrences 8 years after the treatment. 


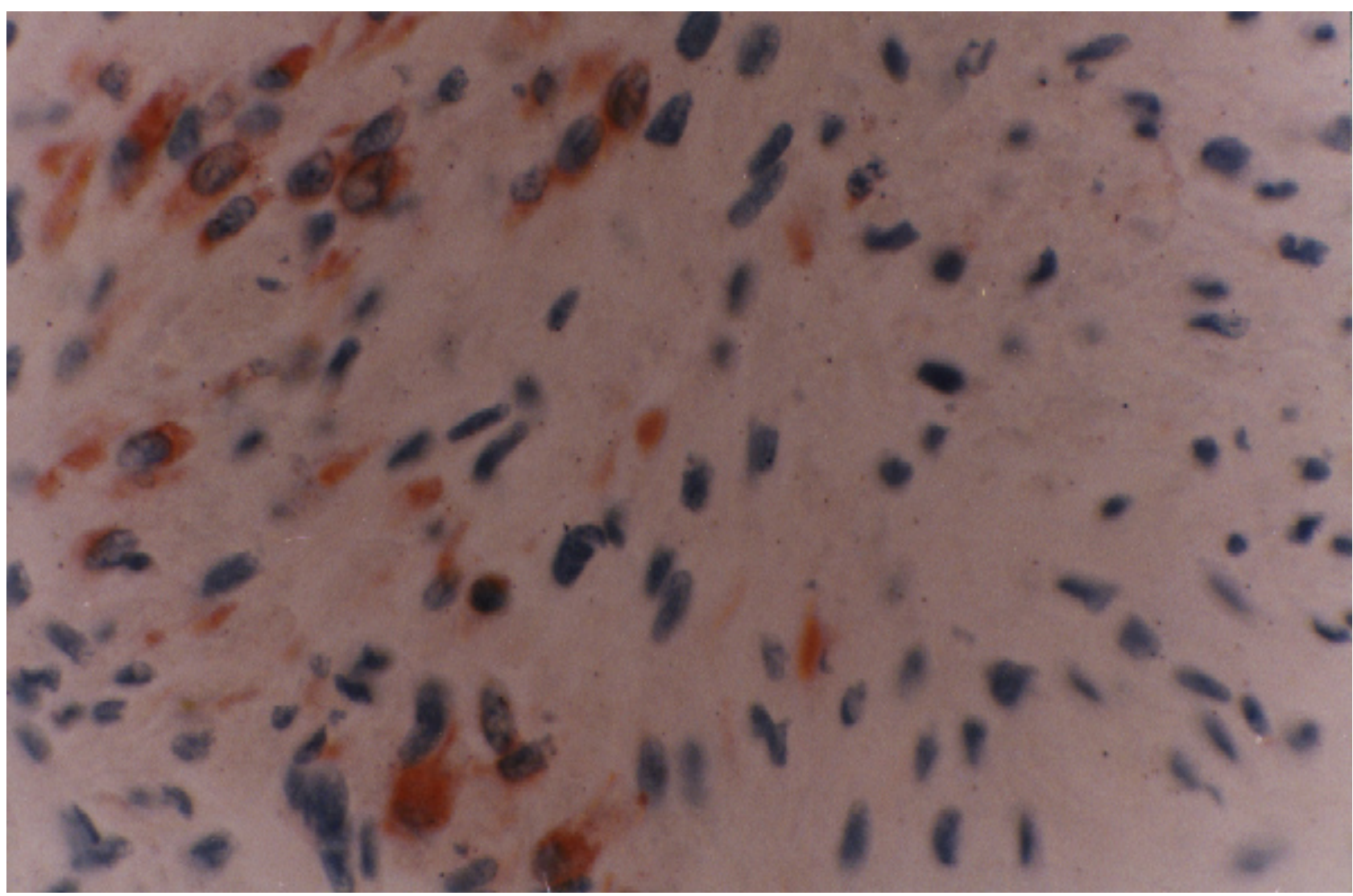

Figure 1. The immunohistochemical stains for $\alpha$-smooth muscle actin are diffusely and strongly positive in the tumor cells.

\section{DISCUSSION}

Sarcomas comprise less than $1 \%$ of all primary breast neoplasms and only a minority of these are leiomyosarcomas [1] Leiomyosarcoma is not rare among soft tissue tumors. It can exist in any part of the body. However, its most common primary sites are the uterus, retroperitoneum, subcutaneous tissues and gastrointestinal tract [3]. Leiomyosarcomas of the breast are extremely rare, either primary or metastatic from other sites. The smooth muscle component might be originated from the blood vessels and sarcomatous change is usually from leiomyoma or other spindle cell tumors [4]. Our presented case should be regarded as primary leiomyosarcoma of the breast since there was no other primary focus being found after staging. Confirming the diagnosis on either IHC or electron microscopy is important as it is sometimes difficult to differentiate these pathologically from poorly differentiated sarcomatoid carcinomas [5].

Diagnosis can be challenging because of the nonspecific clinical and radiological findings. Clinically they present with a progressively enlarging tumour. The presence of enlarged axillary nodes is rare making axillary node staging unnecessary [6]. Our patient had a progr- essively tumor evolving for 17 months, with ipsilateral axillary lymphadenopathy nonspecific.

Radiological findings are also non specific. Leiomyosarcoma is observed as a dense, circumscribed, lobulated mass on mammography, showing features similar to phyllodes tumors or fi broadenomas as described in most reports. Furthermore, on ultrasonography, as reported in all the reports cited, leiomyosarcomas are either oval or lobulated tumors with a smooth clear border, mimicking a fi broadenoma, phyllodes tumor, or an intracystic papilloma. Therefore, with an image analysis alone, differential diagnosis between a leiomyosarcoma and a benign tumor can be extremely diffi cult $[7,8]$.

Histologically, leiomyosarcoma is characterized by interlacing bundles of spindle cells and the absence of ductal or epithelial differentiation. Assessment, Immunohistochemical staining is essential as adjuncts to differentiate leiomyosarcomas from other tumours and soft tissue sarcomas. These tumours are usually positive for desmin, smooth muscle actin, muscle specific actin and negative for neural tumour marker (PS 100), cytokeratins, and epithelial markers. [2,9]

The principal treatment strategy is surgical resection. The complete excision of the tumor by completely including the surgical margin should be recommended as the 
best treatment. Most authors advice either radical or simple mastectomy arguing that wide excision is associated with higher rate of recurrence [2,5]. Though axillary node dissection is not necessary, it has been performed in many of the reported cases because of uncertain preoperative diagnosis or if there were clinically palpable nodes [6]. In our case, the patient underwent a radical mastectomy with lymph node dissection for the presence of clinically palpable axillary lymph nodes, lymph nodes were free of metastases Axillary. There is no evidence describing whether radiation therapy and/or chemotherapy improve the disease-free rate or overall survival [10].

In general, the prognosis of patients with leiomyosarcoma of the breast is considered to be better than that for patients with other sarcomas of the breast [11]

\section{CONCLUSIONS}

Leiomyosarcoma of the breast is extremely rare. To date, the factors that are predictive of patient prognosis have not been identified. Since local recurrences and distant metastases could occur after a relatively long period, a long-term follow-up is necessary.

\section{CONSENT}

Written informed consent was obtained from the patient for publication of this case report.

\section{COMPETING INTERESTS}

The authors declare that they have no competing interests.

\section{REFERENCES}

[1] Abd El-All, H.S. (2006) Breast spindle cell tumours: About eight cases. Diagnostic Pathology, 1, 13. doi:10.1186/1746-1596-1-13

[2] De la Pena, J, Wapnir, I. (2008) Leiomyosarcoma of the breast in a patient with a 10-year-history of cyclophosphamide exposure: A case report. Cases Journal, 1, 301. doi:10.1186/1757-1626-1-301

[3] Cohen, D.T, Oliva, E., Hahn, P.F., Fuller, A.F. Jr., Lee, S.I. (2007) Uterine smooth-muscle tumors with unusual growth patterns: Imaging with pathologic correlation. American Roentgen Ray Society, 188, 246-255. doi:10.2214/AJR.05.1070

[4] Szekely, E., Madaras, L., Kulka, J., Jaraym B., Nagy, L. (2001) Leiomyosarcoma of the female breast. Pathology \& Oncology Research, 7, 151-153. doi:10.1007/BF03032583

[5] Munitiz, V., Rios, A., Canovas, J., et al. (2004) Primitive leiomyosarcoma of the breast: case report and review of the literature. Breast, 13, 72-76. doi:10.1016/j.breast.2003.09.004

[6] Wong, L.-C., Huang, P.-C., Luh, S.-P., Huang, C.-S. (2008) Primary leiomyosarcoma of the nipple-areola complex: Report of a case and review of literature. Journal of Zhejiang University: Science B, 9, 109-113. doi:10.1631/jzus.B0720246

[7] Gupta, R.K. (2007) Needle aspiration cytology and immunohistologic findings in a case of leiomyosarcoma of the breast. Diagnostic Cytopathology, 35, 254-256. doi:10.1002/dc.20618

[8] Jayaram, G., Jayalakshmi, P., Yip, C.H. (2005) Leiomyosarcoma of the breast: Report of a case with fine needle aspiration cytologic, histologic and immunohistochemical features. Acta Cytologica, 49, 656-660. doi:10.1159/000326256

[9] Lee, J., Li, S., Torbenson, M., et al. (2004) Leiomyosarcoma of the breast: A pathologic and comparative genomic hybridization study of two cases. Cancer Genetics and Cytogenetic, 149, 53-57. doi:10.1016/S0165-4608(03)00286-3

[10] Stafyla, V.K., Gauvin, J.M., Farley, D.R. (2004) A 53-year-old woman with a leiomyosarcoma of the breast. Current Surgery, 61, 572-575. doi:10.1016/j.cursur.2004.05.008

[11] Liang, W.C., Sickle-Santanello, B.J., Nims, T.A., et al. (2003) Primary leiomyosarcoma of the breast: A case report with review of the literature, The Breast Journal, 9, 494-496. doi:10.1046/j.1524-4741.2003.09613.x 